\title{
Editorial
}

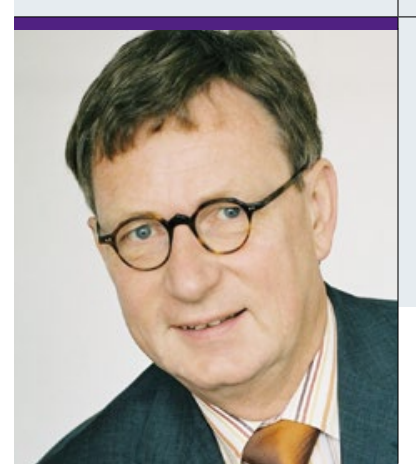

"Das veraltete Embryonenschutzgesetz zwingt

Fortpflanzungsmediziner nicht selten zu einer dem

heutigen internationalen Stand nicht mehr angemessenen

Behandlung, was zu unnötigen Risiken führt."

Prof. Dr. med. Thomas Katzorke

gründete 1981 in Essen die erste Praxis für Reproduktionsmedizin, „novum“

\section{Deutschland braucht ein neues Fortpflanzungsmedizingesetz}

D ie rechtliche Regelung der Fortpflanzungsmedizin ist dringend reformbedürftig. Das Embryonenschutzgesetz von 1990 erfasst die neuesten technischen Entwicklungen nicht, ist in manchen Bereichen unstimmig und lückenhaft, setzt betroffene Frauen, Paare und Kinder unnötigen gesundheitlichen Risiken aus, erschwert die Durchsetzung von Kinderrechten und erzeugt Gerechtigkeitsprobleme und Rechtsunsicherheit für die betroffenen Paare und behandelnden Ärzte [Diskussion Nr. 13 der Leopoldina - Nationale Akademie der Wissenschaften 2017].

Die Fortpflanzungsmedizin entwickelt sich rasant. Immer mehr Personen gründen mithilfe von Techniken der Fortpflanzungsmedizin eine Familie. Pro Jahr werden in Deutschland über 80.000 In-vitroFertilisationen durchgeführt. Weil aus gesellschaftlichen Gründen das Durchschnittsalter der Erstgebärden steigt, aber die biologische Fruchtbarkeit im Alter abnimmt, wird die Zahl der Hilfesuchenden weiter steigen. Zudem werden weltweit Verfahren wie Eizellspende, Embryospende oder Leihmutterschaft zunehmend in Anspruch genommen. Da diese Verfahren wie andere Möglichkeiten der modernen Fortpflanzungsmedizin gewichtige ethische Fragen aufwerfen und wesentliche individuelle Rechte betreffen, bedarf es einer angemessenen rechtlichen Regelung.

Allerdings ist die Rechtslage in Deutschland seit Langem unzureichend. Das Embryonenschutzgesetz ist fast 30 Jahre alt und zwingt Fortpflanzungsmediziner nicht selten zu einer dem heutigen internationalen Stand nicht mehr angemessenen Behandlung, was unnötige Risiken für Mutter und Kind bedeutet. Es wird auch dem gesellschaftlichen Wandel und der Vielfalt heutiger Familienformen nicht mehr gerecht. In Folge einander widersprechender gesetzlicher Regelungen im Embryonenschutzgesetz einerseits und im Familien- und Sozialrecht sowie ärztlichen Berufsrecht andererseits, kommt es zur Ungleichbehandlung von verheirateten und unverheirateten, heterosexuel- len und gleichgeschlechtlichen Paaren sowie alleinstehenden Personen. Die defizitäre Rechtslage führt oft auch zu Nachteilen für das Kind, weil die rechtliche Eltern-Kind-Zuordnung unzureichend geregelt ist und viele Kinder ihr Recht auf Kenntnis der Abstammung nicht durchsetzen können. Viele weitere wichtige Fragen wie die psychosoziale Beratung sind ebenfalls gesetzlich nicht hinreichend geregelt. All dies zeigt, wie notwendig eine neue, umfassende Regelung der Fortpflanzungsmedizin ist. Zwar hat es schon punktuelle Ergänzungen des geltenden Rechts gegeben, zum Beispiel zur eingeschränkten Zulassung der Präimplantationsdiagnostik oder zur Errichtung eines Samenspenderregisters 2016 (vgl. auch Beitrag auf S. 24 ff.). Diese reichen jedoch bei Weitem nicht aus.

Als besonders regelungsbedürftig erweisen sich: — Berücksichtigung des medizinischen Fortschritts im Bereich der Labormethoden; _Zulassung der Eizellspende;

_Zulassung der Embryonenspende;

- Reform des Abstammungsrechts;

- Regelung der Abstammung bei im Ausland durchgeführten Behandlungen, die in Deutschland nicht zulässig sind;

_Detailfragen, wie z.B. Anzahl der Nachkommen pro Spender bei der Samenspende;

- Regelungen zur Kryokonservierung.

Weiterhin muss auf aktuelle Entwicklungen wie die Anwendung von Genscheren wie CRISPR/Cas 9 zur Genomveränderung Stellung bezogen werden. Mögliche Veränderungen der menschlichen Keimbahn müssen breit und intensiv diskutiert werden.

Der neu gewählte Bundestag und die zukünftige Bundesregierung müssen das Thema schnellstmöglich auf die Agenda setzen.

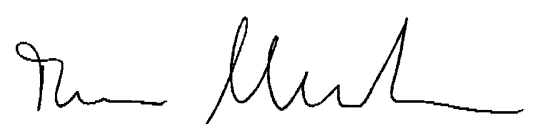

